\title{
Mitochondrial disorder should be considered as a differential of late-onset myasthenia gravis
}

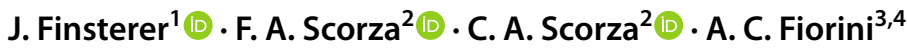

Received: 16 February 2020 / Accepted: 20 March 2020 / Published online: 20 September 2020

(c) Belgian Neurological Society 2020

Keywords Myasthenia $\cdot$ Nonagenarian $\cdot$ Mitochondrial $\cdot$ Repetitive nerve stimulation $\cdot$ Ptosis $\cdot$ Double vision

\section{Commentary}

With interest, we read the article by Oliveria et al. about a 93-year-old female with a 2 months history of generalised weakness, ptosis, double vision, dysarthria, dysphagia, neck pain, and hypophonia, being diagnosed as myasthenia gravis [1]. The authors concluded that the outcome would be more favourable if the diagnosis would have been established earlier. The study has a number of shortcomings.

We do not agree with the conclusions. The outcome was excellent. Missing myasthenia is a frequent event. If the diagnosis would have been established, for instance 4 weeks earlier, the outcome, most likely, would have been the same. Myasthenia is often overlooked for weeks or months before being correctly diagnosed. Nevertheless, it is surprising that myasthenia was not considered earlier in thus 93-year-old

\section{J. Finsterer}

fifigs1@yahoo.de

F. A. Scorza

scorza@unifesp.br

C. A. Scorza

carlascorza.nexp@gmail.com

A. C. Fiorini

acfiorini@pucsp.br

1 Krankenanstalt Rudolfstiftung, Messerli Institute, Postfach 201180 Vienna, Austria

2 Disciplina de Neurociência, Universidade Federal de São Paulo/Escola Paulista de Medicina (UNIFESP/EPM), São Paulo, Brazil

3 Programa de Estudos Pós-Graduado Em Fonoaudiologia, Pontifícia Universidade Católica de São Paulo (PUC-SP), São Paulo, Brazil

4 Departamento de Fonoaudiologia, Escola Paulista de Medicina/Universidade Federal de São Paulo (EPM/UNIFESP), São Paulo, Brazil patient with ptosis, double, vision, dysarthria, dysphagia, and quadruparesis.

Missing are the exact titers of the acetyl-choline receptor antibodies and the appropriate reference limits. Considering that acetyl-choline receptor antibodies were only slightly elevated, differential diagnoses should be discussed, as a decremental response on repetitive nerve stimulation may not only be found in myasthenia gravis and as pyridostigmine may not only be beneficial in myasthenia gravis. Particularly excluded should be a mitochondrial disorder (MID), as the latter may be easily misdiagnosed as myasthenia [2].

Needle electromyography (EMG) in myasthenia gravis patients may not only show a myogenic pattern [1]. Needle EMG using concentric needles may be also normal, non-specifically abnormal, or neurogenic in myasthenia [3, 4]. Since the EMG was myogenic, myopathy should be appropriately excluded. Was the family history positive for neuromuscular disorders?

Missing is an extensive individual history and the current medication the patent was taking prior to the development of myasthenic symptoms. Knowing the current medication is crucial as drugs can strongly influence the clinical presentation and may even trigger the development of myasthenia [5]. Was the drug regimen changed prior to the occurrence of myasthenia?

We should be informed in which nerves repetitive nerve stimulation was carried out, in how many nerves the decremental response was $>10 \%$, and which were the exact values of the decrement. Though thoracic computed tomography was described as normal [1], we should know if a thymoma or thymus hyperplasia was definitively excluded.

Neck pain is an unusual feature of generalised myasthenia. Was it due to head drop or was it unrelated to myasthenia?

Overall, this case report has a number of shortcomings, which need to be addressed before drawing final conclusions. 
Early diagnosis of myasthenia is desirable, but if delayed the outcome is usually not much different than when diagnosed earlier. Given the clinical, blood chemical, and electrophysiological findings, a mitochondrial should be excluded.

Author contribution JF: design, literature search, discussion, first draft, critical comments. All authors have read the journal's position on issues involved in ethical publication.

Funding No funding was received.

\section{Compliance with ethical standards}

Conflict of interest There are no conflicts of interest.

\section{References}

1. Oliveira R, Landeiro L, Lauterbach M (2020) Very late onset of myasthenia gravis: case report and brief review of the literature. Acta Neurol Belg. https://doi.org/10.1007/s13760-020-01290-w
2. Finsterer J (2010) Mitochondrial disorder mimicking ocular myasthenia. Acta Neurol Belg 110:110-112

3. Kannaditharayil D, Napier F, Granit V, Bieri P, Herskovitz S (2017) Abnormal spontaneous activity on needle electromyography in myasthenia gravis. Muscle Nerve 56:E11-E12

4. Samuraki M, Furui E, Komai K, Takamori M, Yamada M (2007) Myasthenia gravis presenting with unusual neurogenic muscle atrophy. Muscle Nerve 36:394-399

5. Berrih-Aknin S (2014) Myasthenia Gravis: paradox versus paradigm in autoimmunity. J Autoimmun 52:1-28

Publisher's Note Springer Nature remains neutral with regard to jurisdictional claims in published maps and institutional affiliations. 